\title{
A NOTE ON INVERSE SYSTEMS OF $S(n)$-CLOSED SPACES
}

\author{
IVAN LONČAR
}

\begin{abstract}
The aim of this paper is to study inverse systems of the $S(n)$-closed spaces which are the generalization of H-closed and Urysohnclosed spaces.
\end{abstract}

\section{INTRODUCTION}

In this paper the symbol $\mathbb{N}^{+}$denotes the set of positive integers and $\mathbb{N}=(0) \cup \mathbb{N}^{+}$.

The concept of $\theta$-closure was introduced by Veličko [16]. For a subset $M$ of a topological space $X$ the $\theta$-closure is defined by $C l_{\theta} M=\{x \in X$ : every closed neighborhood of $x$ meets $M\}, M$ is $\theta$-closed if $C l_{\theta} M=M$. This concept was used by many authors for the study of Hausdorff non-regular spaces. The $\theta$-closure is related especially to Urysohn spaces (every pair of distinct points can be separated by disjoint closed neighborhoods). A space $X$ is Urysohn iff the diagonal in $X \times X$ is $\theta$-closed.

We say that a pair $(G, H)$ is an ordered pair of open sets about $x \in X$ if $G$ and $H$ are open subsets of $X$ and $x \in G \subset C l G \subset H$. A point $x \in X$ is in the $u$-closure of a subset $K \subset X\left(x \in C l_{u} K\right)$ if each ordered pair $(G, H)$ of open sets about $x \in X$ satisfies $K \cap C l H \neq \emptyset$. A subset $K$ of a space $X$ is $u$-closed if $K=C l_{u} K$.

A generalization of the concepts of $\theta$-closure and of $u$-closure is $\theta^{n}$ closure defined in Section 2. Section 3 is the main section of this paper. In this section we study the inverse systems of $\mathrm{S}(\mathrm{n})$-closed spaces. We shall show that an inverse limit of $\mathrm{S}(\mathrm{n})$-closed space and $\Theta^{n}$-closed bonding mapping $p_{a b}$ is non-empty (Theorem 3.5). Moreover, if the projections $p_{a}: \lim \mathbf{X} \rightarrow \mathbf{X}_{a}, a \in A$, are $\Theta^{n}$-closed and the $\theta^{n}$-closure $C l_{\theta^{n}} M$ is Kuratowski Closure Operator i.e. $C l_{\theta^{n}}\left(C l_{\theta^{n}}(A)\right)=C l_{\theta^{n}}(A)$, then $X=\lim \mathbf{X}$ is non-empty and $\mathrm{S}(\mathrm{n})$-closed (Theorem 3.7).

2010 Mathematics Subject Classification. Primary: 54A05, Secondary: 54B35.

Key words and phrases. Inverse systems, S(n)-closed .

Copyright (c) 2015 by ANUBiH. 


\section{2. $\mathrm{S}(\mathrm{N})$-SPACES}

For a positive integer $n$ and a subset $M$ of a topological space $X$, the $\theta^{n}$-closure $C l_{\theta^{n}} M$ of $M$ is defined to be the set [3]

$\{x \in X$ : for every chain of open neighborhoods of $x$,

if $U_{1} \subset U_{2} \subset \cdots \subset U_{n}$ with $C l\left(U_{i}\right) \subset U_{i+1}$, where $i=1,2, \ldots, n-1$, then one has $\left.C l\left(U_{n}\right) \cap M \neq \emptyset\right\}$.

For $n=1$ this gives $\theta$-closure. Moreover, for $n=2$ the above definition gives $u$-closure.

Definition 2.1. A subset $M$ of $X$ is said to be $\theta^{n}$-closed if $M=C l_{\theta^{n}} M$. Similarly $\theta^{n}$-interior of $M$ is defined and denoted by $\operatorname{Int}_{\theta^{n}} M$, so $\operatorname{Int}_{\theta^{n}} M=$ $X \backslash C l_{\theta^{n}}(X \backslash M)$.

Proposition 1. Every $\theta^{n}$ - closed subset $M \subset X$ is closed.

Proof. See [14, p. 222].

Definition 2.2. An open set $U$ is called a $n$-hull of a set $A$ (see [9, p. 624]) if there exists a family of open sets $U_{1}, U_{2}, \ldots, U_{n}=U$ such that $A \subset U_{1}$ and $C l U_{i} \subset U_{i+1}$ for $i=1, \ldots, n-1$.

Definition 2.3. For $n \in \mathbb{N}$ and a filter $\mathcal{F}$ on $X$ we denote by $\operatorname{ad}_{\theta^{n}} \mathcal{F}$ the set of $\theta^{n}$ - adherent points of $\mathcal{F}$, i.e. $\operatorname{ad}_{\theta^{n}} \mathcal{F}=\cap\left\{C l_{\theta^{n}} F_{\alpha}: F_{\alpha} \in \mathcal{F}\right\}$. In particular $\operatorname{ad}_{\theta^{0}} \mathcal{F}=\operatorname{ad} \mathcal{F}$ is the set of adherent points of $\mathcal{F}$.

Definition 2.4. Let $X$ be a space and $n \in \mathbb{N}$; a point $x$ of $X$ is $S(n)$ separated from a subset $M$ of $X$ if $x \notin C l{ }_{\theta^{n}} M$. In particular $x$ is $S(0)$ separated from $M$ if $x \notin C l M$.

Definition 2.5. Let $n \in \mathbb{N}$ and $X$ be a space:

(a) $X$ is an $S(n)$-space if every pair of distinct points of $X$ are $\mathrm{S}(\mathrm{n})$ separated;

(b) A filter $\mathcal{F}$ on $X$ is an $S(n)$-filter if every nonadherent point of $\mathcal{F}$ is $S(n)$-separated from some member of $\mathcal{F}$;

(c) An open cover $\left\{U_{\alpha}\right\}$ of $X$ is an $S(n)$-cover if every point of $X$ is in the $\theta^{n}$-interior of some $U_{\alpha}$.

The $S(n)$-spaces coincide with the $\bar{T}_{n}$-spaces defined in [17] and studied further in [10], where also $S(\alpha)$-spaces are defined for each ordinal $\alpha$.

Proposition 2. The $S(0)$-spaces are the $T_{0}$ spaces, the $S(1)$-spaces are the Hausdorff spaces and the $S(2)$-spaces are the Urysohn spaces.

Clearly every filter is an $S(0)$-filter, every open cover is an $S(0)$-cover and every open filter is an $S(1)$-filter. The open $S(2)$-filters coincide with the 
Urysohn filters defined in [6] and [12]. For $n \geq 1$ the open $S(n)$-filters were defined in [10]. The special covers used in (3.9) [10] are $S(n-1)$ covers, $S(2)$-covers are the Urysohn covers defined in [1]. In a regular space every filter (resp. open cover) is an $S(\mathrm{n})$-filter (resp. $S(n)$-cover) for every $n \in \mathbb{N}$.

The following proposition plays fundamental role in this paper.

Proposition 3. In any topological space:

a) The empty set and the whole space are $\Theta^{n}$-closed,

b) An arbitrary finite union of $\Theta^{n}$-closed sets is $\Theta^{n}$-closed,

c) An arbitrary intersection of $\Theta^{n}$-closed sets is $\Theta^{n}$-closed,

d) $A \Theta^{n}$-closed subset is closed,

e) $C l K \subset C l_{\Theta^{n}} K$ for each subset $K$.

Proof. a) By definition.

b) Let $F=\cup\left\{F_{i}: i=1, \ldots, n\right\}$ where each $F_{i}$ is $\Theta^{n}$-closed. For each $x \notin F$ there exist n-hull $U_{i}$ of $x$ such that $C l U_{i} \cap F_{i}=\emptyset, i=1 \ldots, n$. Now $U=\cap\left\{U_{i}: i=1, \ldots, n\right\}$ is n-hull of $x$ such that $C l U \cap F=\emptyset$. This means that $x \notin C l_{\theta^{n}} F$, i.e. $F$ is $\Theta^{n}$-closed.

c) Assume that $x \in C l_{\theta^{n}} F$, where $F=\cap\left\{F_{\alpha}: \alpha \in A\right\}$ and each $F_{\alpha}$ is $\theta^{n}$ - closed. This means that for each n-hull $U$ of the point $x$ we have $C l U \cap F \neq \emptyset$. Clearly $C l U \cap F_{\alpha} \neq \emptyset$ for every $\alpha \in A$. We infer that $x \in F_{\alpha}$, $\alpha \in A$, since each $F_{\alpha}$ is $\theta^{n}$ - closed. Finally, $x \in \cap\left\{F_{\alpha}: \alpha \in A\right\}=F$ and $F$ is $\theta^{n}$ - closed $\left(F=C l_{\theta^{n}} F\right)$.

d) See Proposition 1.

e) The set $C l K$ is the minimal closed set containing $K$. Hence, $C l K \subset$ $C l_{\Theta^{n}} K$.

From (a) and (b) we get the following.

Lemma 2.1. If $X$ is a topological space, then for each $Y \subset X$ there exists a minimal $\Theta^{n}$-closed subset $Z \subset X$ such that $Y \subset Z$.

Proof. The collection $\Phi$ of all $\Theta^{n}$-closed subsets $W$ of $X$ which contains $Y$ is non-empty since $X \in \Phi$. By (b) of Proposition 3 we infer that $Z=\cap\{W$ : $W \in \Phi\}$ is a minimal $\Theta^{n}$-closed subset $Z \subset X$ containing $Y$.

Proposition 4. If $n \geq 1$, then every $\theta^{n}$ - closed subset $M \subset X$ is $\theta$ - closed.

Proof. If $n=1$, then $\theta^{n}$-closure gives the $\theta$-closure. Suppose that $n \geq 2$ and that some $\theta^{n}$ - closed subset $M \subset X$ is not $\theta$ - closed. This means there exists a point $x \in X \backslash M$ such that for every open set $U$ which contains $x$ the set $C l U \cap M$ is non-empty. But $x$ is not in $\theta^{n}$ - closure of $M$, thus there exists a chain of open neighborhoods of $x, U_{1} \subset U_{2} \subset \cdots \subset U_{n}$, such that $C l U_{i} \subset U_{i+1}$ for $i=1,2, \ldots, n-1$ and $C l U_{n} \cap M=\emptyset$. This means that $U_{n}$ is the neighborhood of $x$ such that $C l U_{n} \cap M=\emptyset$. This contradicts 
the fact that for every open set $U$ which contains $x$ the set $C l U \cap M$ is non-empty.

Definition 2.6. For a space $(X, \tau)$ and $n \in \mathbb{N}$ denote by $\left(X, \tau_{\theta^{n}}\right)$, where $\tau_{\theta^{n}}$ is the topology on $X$ generated by the $\theta^{n}$-closure, i.e. having as closed sets all $\theta^{n}$-closed sets in $(X, \tau)$.

Clearly $\tau_{\theta^{0}}=\tau, \tau_{\theta^{1}}=\tau_{\theta}$ and a subset $U$ of $X$ is $\tau_{\theta^{n} \text {-open iff every element }}$ of $U$ is contained in the $\theta^{n}$-interior of $U$.

The next proposition follows directly from the definitions.

Proposition 5. For a topological space $(X, \tau)$ and $n \in \mathbb{N}^{+}$the following conditions are equivalent:

(a) $(X, \tau)$ is an $S(n)$-space,

(b) $\left(X, \tau_{\theta^{n}}\right)$ is a $T_{1}$ space

(c) $\left(X, \tau_{\theta^{n}}\right)$ is $T_{0}$ and $(X, \tau)$ is $T_{1}$.

If $n \geq 1$, then these conditions are equivalent to:

(d) $\left(X, \tau_{\theta^{n}}\right)$ is $T_{0}$.

If $n=2 k$ with $k \in \mathbb{N}^{+}$, then the above conditions are equivalent to:

(e) The diagonal in $X \times X$ is $\theta^{k}$-closed

This proposition show the pivotal rôle of the topologies $\tau_{\theta^{k}}$ in the study of $S(n)$-spaces for $n>1$. In some sense they replace the semiregularization which was the main tool in the study of $H$-closed spaces.

Let us observe that we have the following result.

Proposition 6. The identity mapping $i:(X, \tau) \rightarrow\left(X, \tau_{\theta^{n}}\right)$ is continuous.

Definition 2.7. Let $f:(X, \tau) \rightarrow(Y, \sigma)$ be a mapping. We define a mapping $f_{\Theta^{n}}:\left(X, \tau_{\theta^{n}}\right) \rightarrow\left(Y, \sigma_{\theta^{n}}\right)$ by $f_{\Theta^{n}}(x)=f(x)$ for every $x \in X$, i.e., the following diagram

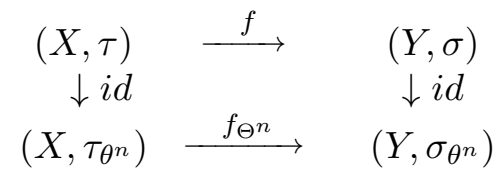

commutes.

Lemma 2.2. The mapping $f_{\Theta^{n}}:\left(X, \tau_{\theta^{n}}\right) \rightarrow\left(Y, \sigma_{\theta^{n}}\right)$ is continuous.

Proof. Let us prove that $f_{\Theta^{n}}^{-1}(F)$ is closed in $\left(X, \tau_{\theta^{n}}\right)$ if $F$ is closed in $\left(Y, \sigma_{\theta^{n}}\right)$. It suffices to prove that $f^{-1}(F)$ is $\Theta^{n}$-closed in $X$ if $F$ is $\Theta^{n}$-closed in $Y$. If $x \in X \backslash f^{-1}(F)$, then $f(x) \notin F$. There exists an open set $U$ such that $f(x) \in U$ and $C l U \cap F=\emptyset$ since $F$ is $\Theta^{n}$-closed in $Y$. The open set $f^{-1}(U)$ contains $x$ and $C l f^{-1}(U) \cap f^{-1}(F)=\emptyset$ since $f^{-1}(C l U) \cap f^{-1}(F)=\emptyset$. Hence, if $x \in X \backslash f^{-1}(F)$, then $x \in X \backslash C l_{\Theta^{n}} f^{-1}(F)$, and, consequently, $f^{-1}(F)$ is $\Theta^{n}$-closed in $X$. 
Proposition 7. For every $M \subset X$ it follows

$$
\operatorname{cl}_{\theta^{n}} M=\cap(C l U: U \text { is } n \text {-hull of } M) .
$$

Proof. a) Let us prove that $c l_{\theta^{n}} M \subset \cap(C l U: U$ is $n$-hull of $M)$. If $x \in c l_{\theta^{n}} M$ then it is impossible that $x \notin \cap(C l U: U$ is $n$-hull of $M)$ since then there exists $n$-hull $U$ of $M$ such that $x \notin C l U$. This means that there exists a family of open sets $U_{1}, U_{2}, \ldots, U_{n}=U$ such that $A \subset U_{1}$ and $C l U_{i} \subset U_{i+1}$ for $i=1, \ldots, n-1$. Now, $V_{1}=X \backslash C l U_{1} \supset V_{2}=X \backslash C l U_{2} \supset \cdots \supset V_{n}=$ $X \backslash C l U_{n}$ is the open sets such that $x \in V_{n} \subset V_{n-1} \subset \cdots \subset V_{1}$ which satisfy the Definition 2.2. Hence, $x \notin c l_{\theta^{n}} M$, a contradiction.

b) Let us prove that

$$
\operatorname{cl}_{\theta^{n}} M \supset \cap(C l U: U \text { is } n \text {-hull of } M) .
$$

Suppose that $x \in \cap\{C l U: U$ is $n$-hull of $M\}$ is not in $\operatorname{cl}_{\theta^{n}} M$, i.e. $x \notin$ $c l_{\theta^{n}} M$. By the Definition 2.2 there exists a chain of open neighborhoods of $x, U_{1} \subset U_{2} \subset \cdots \subset U_{n}$, such that $C l U_{i} \subset U_{i+1}$ for $i=1,2, \ldots, n-1$ and $C l U_{n} \cap M=\emptyset$. Now, a chain of open neighborhoods of $M, V_{1}=X \backslash C l U_{1} \supset$ $V_{2}=X \backslash C l U_{2} \supset \cdots \supset V_{n}=X \backslash C l U_{n}$ is a n-hull $V=V_{1}$ of $M$ such that $x \notin C l V_{1}$ since $C l V_{1} \cap U_{1}=\emptyset$. The proof of $c l_{\theta^{n}} M \supset \cap(C l U: U$ is $n$-hull of $M)$ is completed.

Corollary 2.3. A subset $M$ of the space $X$ is $\theta^{n}$ - closed if and only if

$$
M=\cap(C l U: U \text { is } n \text {-hull of } M) .
$$

Definition 2.8. A mapping $f: X \rightarrow Y$ is said to be $\Theta^{n}$-closed if $f(F)$ is $\Theta^{n}$-closed for each $\Theta^{n}$-closed subset $F \subset X$.

Lemma 2.4. Let $f: X \rightarrow Y$ be a continuous mapping. The following conditions are equivalent:

(a) $f$ is $\theta^{n}$-closed,

(b) For every $B \subset Y$ and each $\Theta^{n}$-open set $U \supseteq f^{-1}(B)$ there exists a $\Theta^{n}$-open set $V \supseteq B$ such that $f^{-1}(V) \subset U$.

(c) $f_{\theta^{n}}$ is a closed mapping.

Proof. The proof is similar to the proof of the corresponding theorem for closed mappings [4, p. 52].

Question. Let $f: X \rightarrow Y$ be a mapping. Under what conditions $f$ is $\theta^{n}$-closed?

Definition 2.9. Following [2, p. 48, (31)] we say that $f: X \rightarrow Y$ is $\Theta^{n}$-perfect if and only if for every filter base $\mathcal{F}$ on $X$

$$
f\left(\cap\left\{c 1_{\theta^{n}} F: F \in \mathcal{F}\right\}\right) \supseteq \cap\left\{c 1_{\theta^{n}} f(F): F \in \mathcal{F}\right\}
$$

Lemma 2.5. If $f: X \rightarrow Y$ is $\Theta^{n}$-perfect, then: 
(a) For each $A \subseteq X, c 1_{\theta^{n}} f(A) \subseteq f\left(\mathrm{c} 1_{\theta^{n}} A\right\rangle$.

(b) For each $\theta^{n}$-closed $\mathrm{A} \subseteq X, f(\mathrm{~A})$ is $\theta^{n}$-closed.

Definition 2.10. A function $f: X \rightarrow Y$ is almost $\Theta^{n}$-closed if for any set $A \subseteq X, f\left(\mathrm{c} 1_{\theta} A\right)=\mathrm{c} 1_{\theta} f(\mathrm{~A})$.

Lemma 2.6. If $f: X \rightarrow Y$ is almost $\Theta^{n}$-closed, then it is $\Theta^{n}$-closed.

Definition 2.11. A mapping $f: X \rightarrow Y$ is said to be skeletal $(H J)[7, \mathrm{p}$. 13] if for each open (regularly open) subset $U \subset X$ we have $\operatorname{Int} f^{-1}(C l U) \subset$ $\mathrm{Clf}^{-1}(U)$.

A mapping $f: X \rightarrow Y$ is said to have the inverse property if $C l f^{-1}(U)=$ $f^{-1}(C l U)$ for every open set $U$ in $Y$.

Each open mapping has the inverse property since a mapping $f: X \rightarrow Y$ is open if and only if $f^{-1}(C l B)=C l f^{-1}(B)$ or - equivalently - Int $f^{-1}(B)=$ $f^{-1}(\operatorname{Int} B)$ for every $B \subset Y$ [4, Exercise 1.4.C, p. 57].

Proposition 8. [7, p. 13]. A mapping $f: X \rightarrow Y$ is HJ if and only if the counterimage of the boundary of each regularly open set is nowhere dense.

A mapping $p: Y \stackrel{\text { onto }}{\longrightarrow} X$ is said to be irreducible if for each closed subset $A$ of $Y \quad A \neq Y$ implies $C l p(A) \neq X$. A mapping $f: X \rightarrow Y$ is said to be semi-open provided $\operatorname{Int} f(U) \neq \emptyset$ for each non-empty open $U \subset X$. From Proposition 8 it follows the following result (see [7, 1.1, p. 27], [15, p. 236]).

Lemma 2.7. Each semi-open, each open and each closed irreducible mapping is $H J$.

Proposition 9. Let $f: X \rightarrow Y$ be a $\Theta^{n}$-closed mapping and let $F$ be a $\Theta^{n}$-closed subset of $X$. The restriction $g=f \mid F$ is $\Theta^{n}$-closed.

Proof. By (c) of Lemma 2.4 it suffices to prove that $f_{\Theta^{n}} \mid i(F)$ is a closed mapping. Lemma follows since the restriction of closed e mapping $f_{\theta^{n}}$ onto a closed subset is closed.

Lemma 2.8. Let $f: X \rightarrow Y$ be a surjective mapping. If $F$ is $\Theta^{n}$-closed in $Y$, then $f^{-1}(F)$ is $\Theta^{n}$-closed in $X$.

Proof. Let us prove that $X \backslash f^{-1}(F)$ is $\Theta^{n}$-open. If $x$ is a point of $X \backslash f^{-1}$ $(F)$, then $f(x) \in Y \backslash F$. There exists an open hull $U$ such that $f(x) \in U$ and $C l U \cap F=\emptyset$ since $F$ is $\Theta$-closed. Now $x \in f^{-1}(U)$ and $C l f^{-1}(U) \cap F=$ $\emptyset$. We infer that $X \backslash f^{-1}(F)$ is $\Theta^{n}$-open. Hence, $f^{-1}(F)$ is $\Theta^{n}$-closed by Definition 2.1.

Definition 2.12. An $\mathrm{S}(\mathrm{n})$-space $M, n>0$, is $S(n)$-closed ([3]) if it is $\theta^{n}$ closed in every $\mathrm{S}(\mathrm{n})$-space in which it can be embedded. 
Porter and Votaw [10] characterized $S(n)$-closed spaces by means of open $S(n)$-filters and $S(n)$-covers (for $n=2$ it was done by Herrlich [6]). On the other hand Hamlett [5] proved that a Hausdorff space $X$ is $H$-closed iff for every filter $\mathcal{F}$ on $X \operatorname{ad}_{\theta} \mathcal{F} \neq \emptyset$.

Proposition 10. [3, Proposition 2.1., p. 63] Let $n \in \mathbb{N}^{+}$and $X$ be a space. Then the following conditions are equivalent:

(a) For every open filter $\mathcal{F}$ on $X a_{\theta^{n}} \mathcal{F} \neq \emptyset$;

(b) For every filter $\mathcal{F}$ on $X a d_{\theta^{n}} \mathcal{F} \neq \emptyset$;

(c) For every open $S(n)$-filter $\mathcal{F}$ on $X$ ad $\mathcal{F} \neq \emptyset$;

(d) For every $S(n-1)$-cover $\left\{U_{\alpha}\right\}$ of $X$ there exist $\alpha_{l}, \alpha_{2}, \ldots, \alpha_{l \iota}$ such that $X=\bigcup_{i=1}^{k} C l U_{\alpha_{1}}$.

If $X$ is an $S(n)$-space then the above conditions are equivalent to:

(e) $X$ is $S(n)$-closed.

Proposition 11. Let $X$ be $S(n)$-closed space. Let $f: X \rightarrow Y$ be an $H J$ mapping and let $F$ be a $\Theta^{n}$-closed subset $F \subset X$. Then $f(F)$ is $\Theta^{n}$-closed subset $Y$.

Proof. Step 1. By Corollary 2.3 we have

$$
F=\cap(C l U: U \text { is } n \text {-hull of } F,
$$

and let $\mathcal{V}=\left\{V_{\lambda}: \lambda \in \Lambda\right\}$ be a maximal family of n-hull containing $F$. Moreover, let $\mathcal{U}=\left\{U_{\mu}: \mu \in \Omega\right\}$ be a family of all n-hull of $f(F)$ such that there exists $V_{\lambda} \in \mathcal{V}$ such that $f\left(V_{\lambda}\right) \subset U_{\mu}$. For every n-hull $W \ni y$ we have $C l W \cap f\left(V_{\lambda}\right) \neq \emptyset$ since $C l W \cap f\left(V_{\lambda}\right)=\emptyset$ implies $Y \backslash C l W \supset f\left(V_{\lambda}\right)$, $Y \backslash C l W \in \mathcal{U}$ and $y \in C l(Y \backslash C l W)$.

Step 2. Now, the set $W^{*}=\operatorname{Int} C l W$ is regularly open and, by virtue of Definition 2.11, we have

$$
\operatorname{Int} f^{-1}\left(C l W^{*}\right) \subset C l f^{-1}\left(W^{*}\right) .
$$

From this and $f^{-1}\left(C l W^{*}\right) \cap V_{\lambda} \neq \emptyset$ it follows that $f^{-1}\left(W^{*}\right) \cap V_{\lambda} \neq \emptyset$ for each $V_{\lambda} \in \mathcal{V}$. The family $\mathcal{V}^{*}=\left\{V_{\lambda}^{*}: V_{\lambda}^{*}=f^{-1}\left(W^{*}\right) \cap V_{\lambda}\right\}$ has the finite intersection property. From the $\mathrm{S}(\mathrm{n})$-closedness (Proposition 10) of $X$ it follows that there exists adherent point $x \in \cap\left\{C l_{\theta_{n}} V_{\lambda}^{*}: V_{\lambda}^{*} \in \mathcal{V}^{*}\right\}$. It is easy to prove that $x \in F$ and $f(x) \in \cap\{C l W: W$ is open set containing $y\}$. This means that $y=f(x)$ since $Y$ is a Hausdorff space. Hence, $f(F) \supset \operatorname{cl}_{\theta^{n}} f(F)=\cap(C l V: V$ is $n$-hull of $f(F)$. The proof of Proposition is completed.

Corollary 2.9. Each semi-open (open, closed irreducible) and each mapping with the inverse property is $\Theta^{n}$-closed. 
Lemma 2.10. If $X$ is $S(n)$-closed, then every family $\left\{A_{\mu}, \mu \in \Omega\right\}$ of $\theta^{n}$ closed subsets of $X$ with the finite intersection property has a non-empty intersection $\cap\left\{A_{\mu}, \mu \in \Omega\right\}$.

Proof. Let $X$ be S(n)-closed and let $\left\{A_{\mu}, \mu \in \Omega\right\}$ be a family of $\theta^{n}$-closed subsets of $X$ with the finite intersection property. By (b) of Proposition 10 we infer that $\operatorname{ad}_{\theta^{n}}\left\{A_{\mu}, \mu \in \Omega\right\} \neq \emptyset$, i.e. $\cap\left\{C l_{\theta^{n}} A_{\mu}, \mu \in \Omega\right\} \neq \emptyset$ 2.3. But $C l_{\theta^{n}} A_{\mu}=A_{\mu}$ since $\left\{A_{\mu}, \mu \in \Omega\right\}$ is a family of $\theta^{n}$-closed subsets of $X$. Finally we infer that $\cap\left\{A_{\mu}, \mu \in \Omega\right\} \neq \emptyset$.

Lemma 2.11. If $(X, \tau)$ is $S(n)$-closed space then the space $\left(X, \tau_{\theta^{n}}\right)$ is quasi-compact.

Proof. Let $(X, \tau)$ be an $S(n)$-closed space and let us prove that $\left(X, \tau_{\theta^{n}}\right)$ is quasi-compact. For every filter $\mathcal{F}=\{F: F \in \mathcal{F}\}$ of closed sets on $\left(X, \tau_{\theta^{n}}\right)$ we have the family $\left\{C l_{\Theta_{n}} i^{-1}(F): F \in \mathcal{F}\right\}$ with non-empty intersection $\cap\left\{C l_{\Theta_{n}} i^{-1}(F): F \in \mathcal{F}\right\}$ since $(X, \tau)$ is $S(n)$-closed space. It is clear that $\cap\{F: F \in \mathcal{F}\} \neq \emptyset$ since $i\left(C l_{\Theta_{n}} i^{-1}(F)\right)=C l F=F$. Hence, $\left(X, \tau_{\theta^{n}}\right)$ is quasi-compact.

Following Porter and Thomas [11] (for $n=1$ they introduced quasi-Hclosed spaces) the spaces satisfying the equivalent conditions $(a)-(d)$ will be called quasi $S(n)$-closed.

An open set $U$ of a topological space $(X, \tau)$ is regularly open if $U=\operatorname{Int} C l$ $U$. The topology on $X$ which has as a basis the set of regularly open sets of $(X, \tau)$ is denoted by $\tau_{s}$; it is the semiregularization of $\tau$ and $(X, \tau)$ is semiregular if $\tau_{s}=\tau$.

The set $F \subset X$ regularly closed if $F=C l \operatorname{Int} F$. A space $X$ is almost regular if for each regularly closed set $F \subset X$ and each point $x \in X \backslash F$ there are disjoint open sets $U \ni x$ and $V \supset F$. Moreover, the space $(X, \tau)$ is almost regular if $\left(X, \tau_{s}\right)$ is regular.

For a space $X$ and $n \in \mathbb{N}^{+}$denote by $o_{n}(X)$ the (ordinal) number of iterations of the $\theta^{n}$-closure to get a Kuratowski operator (it will be the closure in $\left.\tau_{\theta^{\prime \prime}}\right)$. We call $o_{n}(X) 274^{n}$-order of $X$. By Theorem 1.2 of [3] $o_{1}(X)=1$ iff $X$ is almost regular. We shall sometimes need this result that in an almost regular space $X, C l_{\Theta} A$ is $\Theta$-closed (i.e. $C l_{\Theta}\left(C l_{\Theta} A\right)=C l_{\Theta} A$ [8].

Let $X$ be a set and $\mathcal{P}(X)$ its power set. A Kuratowski Closure Operator is an assignment $c l: \mathcal{P}(X) \rightarrow \mathcal{P}(X)$ with the following properties:

1. $\operatorname{cl}(\emptyset)=\emptyset$ (Preservation of Nullary Union);

2. $A \subset \operatorname{cl}(A)$ (Extensivity);

3. $\operatorname{cl}(A \cup B)=\operatorname{cl}(A) \cup \operatorname{cl}(B)$ (Preservation of Binary Union);

4. $\operatorname{cl}(\operatorname{cl}(A))=\operatorname{cl}(A)$ (Idempotence). 
If the last axiom, Idempotence, is omitted, then the axioms define a Preclosure Operator. It is clear that $\theta^{n}$-closure $C l_{\theta^{n}} M$ is a preclosure operator.

A consequence of the third axiom is: $A \subseteq B \Longrightarrow \operatorname{cl}(A) \subseteq \operatorname{cl}(B)$ (Preservation of Inclusion).

Clearly, (4) implies the following result.

Theorem 2.12. Let $(X, \tau)$ be $S(n)$-space. If $\theta^{n}$-closure $C l_{\theta^{n}} M$ is $K u$ ratowski Closure Operator i.e. $C l_{\theta^{n}}\left(C l_{\theta^{n}}(A)\right)=C l_{\theta^{n}}(A)$, then $(X, \tau)$ is $S(n)$-closed space if and only if the space $\left(X, \tau_{\theta^{n}}\right)$ is quasi-compact.

Proof. The "if" part. Let the space $\left(X, \tau_{\theta^{n}}\right)$ be quasi-compact. For every filter $\mathcal{F}$ on $X$ we have the family $\left\{C l_{\Theta_{n}} F: F \in \mathcal{F}\right\}$. Using the mapping $i:(X, \tau) \rightarrow\left(X, \tau_{\theta^{n}}\right)$ from Proposition 6 we obtain the family $\left\{i\left(C l_{\Theta_{n}} F\right)\right.$ : $F \in \mathcal{F}\}$ which is the filter of closed sets in $\left(X, \tau_{\theta^{n}}\right)$. From the quasicompactness of $\left(X, \tau_{\theta^{n}}\right)$ it follows that $\cap\left\{i\left(C l_{\Theta_{n}} F\right): F \in \mathcal{F}\right\} \neq \emptyset$. Clearly, $\cap\left\{C l_{\Theta_{n}} F: F \in \mathcal{F}\right\}=\operatorname{ad}_{\theta^{n}} \mathcal{F} \neq \emptyset$ (see Definition 2.3). By (e) of Proposition 10 we infer that $(X, \tau)$ is $S(n)$-closed space.

The "only if" part. See 2.11.

Theorem 2.13. $S(n)$-closed space $(X, \tau)$ is quasi-compact if every closed subset of $(X, \tau)$ is $\theta^{n}$ - closed.

Proof. The identity mapping $i:(X, \tau) \rightarrow\left(X, \tau_{\theta^{n}}\right)$ is continuous by Proposition 6 . If every closed subset of $(X, \tau)$ is $\theta^{n}$ - closed, then the identity $i$ is closed. Thus, $i:(X, \tau) \rightarrow\left(X, \tau_{\theta^{n}}\right)$ is the homeomorphism. This means that $(X, \tau)$ is quasi-compact since $\left(X, \tau_{\theta^{n}}\right)$ is quasi-compact by Theorem 2.12 .

\section{InVERSE Systems of $\mathrm{S}(\mathrm{N})$-Closed SPACES - INVERSE System $\mathbf{X}_{\Theta^{n}}$}

This section is the main section of this paper. We shall use the notion of inverse systems as in the book [4, pages 135-144] and we start with the following elementary proposition.

Proposition 12. Let $\mathbf{X}=\left\{X_{a}, p_{a b}, A\right\}$ be an inverse system of $S(n)$-spaces. If the projections $p_{a}: \lim \mathbf{X} \rightarrow \mathbf{X}_{a}, a \in A$, are surjections, then $\lim \mathbf{X}$ is $S(n)$-space.

Proof. Let $x, y$ be a pair of different points in $\lim \mathbf{X}$. There exists an $a \in A$ such that $p_{b}(x) \neq p_{b}(y)$ for every $b \geq a$. By virtue of (a) Definition 2.5 it suffices to prove that every pair of distinct points of $X$ are $\mathrm{S}(\mathrm{n})$-separated (Definition 2.4). Let us prove that $x$ is $\mathrm{S}(\mathrm{n})$-separated from $y$, i,e., that $x$ $\notin C l \theta_{\theta^{n}}\{y\}$. From the fact that $X_{a}$ is $\mathrm{S}(\mathrm{n})$-space it follows that $p_{a}(x)$ is $\mathrm{S}(\mathrm{n})$-separated from $p_{a}(y)$. This means that there exists a n-hull $U 2.2$ of $p_{a}(x)$, i.e., there exists a family of open sets $U_{1}, U_{2}, \ldots, U_{n}=U$ such that 
$p_{a}(x) \in U_{1}, C l U_{i} \subset U_{i+1}$ for $i=1, \ldots, n-1$ and $p_{a}(y) \notin C l U$. It is clear that $p_{a}^{-1}\left(U_{1}\right), p_{a}^{-1}\left(U_{2}\right), \ldots, p_{a}^{-1}\left(U_{n}\right)=p_{a}^{-1}(U)$ is the n-hull of $x$ and $y \notin C l p_{a}^{-1}(U)$. Hence $\lim \mathbf{X}$ is a $\mathrm{S}(\mathrm{n})$-space.

Theorem 3.1. Let $\mathbf{X}=\left\{X_{a}, p_{a b}, A\right\}$ be an inverse system with $H J$ mappings $p_{a b}$. If the projections $p_{a}: \lim \mathbf{X} \rightarrow X_{a}, a \in A$, are surjections, then they are HJ mapping and, consequently, $\Theta^{n}$-closed .

Proof. By Proposition 11 a mapping $f: X \rightarrow Y$ is $H J$ if and only if the counterimage of the boundary of each regularly open set is nowhere dense. Suppose that $p_{a}$ is not $H J$. Then there exist a regularly open set $U_{a}$ in $X_{a}$ such that the boundary of $p_{a}^{-1}\left(U_{a}\right)$ contains an open set $U$. From the definition of a base in $\lim \mathbf{X}$ it follows that there is a $b \geq a$ and an open set $U_{b}$ in $X_{b}$ such that $p_{b}^{-1}\left(U_{b}\right) \subset U$. It is clear that $U_{b} \subset B d p_{a b}^{-1}\left(U_{a}\right)$. This is impossible since $p_{a b}$ is $H J$. Hence, the projections $p_{a}, a \in A$, are HJ. From Proposition 11 it follows that $p_{a}$ is $\Theta^{n}$-closed.

For every inverse system $\mathbf{X}=\left\{X_{a}, p_{a b}, A\right\}$ of $\mathrm{S}(\mathrm{n})$-closed spaces we shall introduce the inverse system $\mathbf{X}_{\Theta^{n}}$. Namely, for every space $X_{a}$ there exists the space $\left(X_{a}\right)_{\Theta^{n}}$ which is defined in Definition 2.6 as the space $\left(X, \tau_{\theta^{n}}\right)$. Moreover, for every mapping $p_{a b}: X_{b} \rightarrow X_{a}$ there exists the mapping $\left(p_{a b}\right)_{\Theta^{n}}$ (see Definition 2.7 and Lemma 2.2). The transitivity condition

$$
\left(p_{a b}\right)_{\Theta^{n}}\left(p_{b c}\right)_{\Theta^{n}}=\left(p_{a c}\right)_{\Theta^{n}}
$$

follows from the commutativity of the diagram 2.1. This means that we have the following result.

Proposition 13. For every inverse system $\mathbf{X}=\left\{X_{a}, p_{a b}, A\right\}$ of $S(n)$-closed spaces there exists the inverse system $\mathbf{X}_{\Theta^{n}}=\left\{\left(X_{a}\right)_{\Theta^{n}},\left(p_{a b}\right)_{\Theta^{n}}, A\right\}$ such that the following diagram commutes

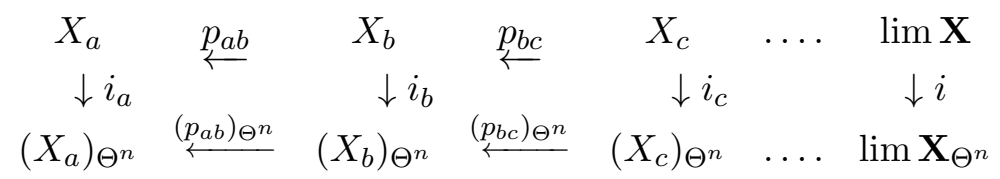

where $i$ and each $i_{a}$ is the identity for every $a \in A$.

Let us recall that the continuity of the mapping $i$ was proved in Proposition 6 .

Proposition 14. Let $\mathbf{X}=\left\{X_{a}, p_{a b}, A\right\}$ be an inverse system. There exists a mapping $p_{\Theta}:(\lim \mathbf{X})_{\Theta^{n}} \rightarrow \lim \mathbf{X}_{\Theta^{n}}$ such that $i=p_{\Theta} i_{\Theta}$, where $i_{\Theta}$ : $\lim \mathbf{X} \rightarrow(\lim \mathbf{X})_{\Theta^{n}}$ is the identity.

Proof. By Definition 2.1 for each $a \in A$ there is $\left(p_{a}\right)_{\Theta}:(\lim \mathbf{X})_{\Theta} \rightarrow\left(X_{a}\right)_{\Theta}$. This mapping is continuous (Lemma 2.2). The collection $\left\{\left(p_{a}\right)_{\Theta}: a \in A\right\}$ 
induces a continuous mapping $p_{\Theta}:(\lim \mathbf{X})_{\Theta} \rightarrow \lim \mathbf{X}_{\Theta}$. Hence we have the following diagram.

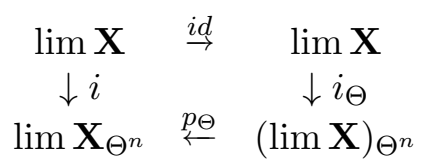

In the sequel we shall use the following results.

Theorem 3.2. [13, Theorem 3, p. 206]. Let $\mathbf{X}=\left\{X_{a}, p_{a b}, A\right\}$ be an inverse system of quasi-compact non-empty $T_{0}$ spaces and closed bonding mapping $p_{a b}$. Then $\lim \mathbf{X}$ is non-empty.

Theorem 3.3. [13, Theorem 5, p. 208].Let $\mathbf{X}=\left\{X_{a}, p_{a b}, A\right\}$ be an inverse system of quasi-compact $T_{0}$ spaces and closed bonding mapping $p_{a b}$. Then $\lim \mathbf{X}$ is quasi-compact.

Now, we shall prove the following result.

Lemma 3.4. Let $\mathbf{X}=\left\{X_{a}, p_{a b}, A\right\}$ be an inverse system of quasi-compact non-empty $T_{0}$ spaces and closed surjective bonding mapping $p_{a b}$. Then the projections $p_{a}: \lim \mathbf{X} \rightarrow \mathbf{X}_{a}, a \in A$, are surjective and closed.

Proof. Let us prove that the projections $p_{a}$ are surjective. For each $x_{a} \in X_{a}$ the sets $Y_{b}=p_{a b}^{-1}\left(x_{a}\right)$ are non-empty closed sets. This means that the system $\mathbf{Y}=\left\{Y_{b}, p_{b c} \mid Y_{c}, a \leq b \leq c\right\}$ satisfies Theorem 3.2 and has a non-empty limit. For every $y \in Y$ we have $p_{a}(y)=x_{a}$. Hence, $p_{a}$ is surjective. It remains to prove that $p_{a}$ is closed. It suffices to prove that for every $x_{a} \in X_{a}$ and every neighborhood $U$ of $p_{a}^{-1}\left(x_{a}\right)$ in $\lim \mathbf{X}$ there exists an open set $U_{a}$ containing $x_{a}$ such that $p_{a}^{-1}\left(U_{a}\right) \subset U$. For every $x \in p_{a}^{-1}\left(x_{a}\right)$ there is a basic open set $p_{a(x)}^{-1}\left(U_{a(x)}\right)$ such that $x \in p_{a(x)}^{-1}\left(U_{a(x)}\right) \subset U$. From the quasicompactness of $p_{a}^{-1}\left(x_{a}\right)$ it follows that there exists a finite set $\left\{x_{1}, \ldots, x_{n}\right\}$ of the points of $p_{a}^{-1}\left(x_{a}\right)$ such that $\left\{p_{a\left(x_{1}\right)}^{-1}\left(U_{a\left(x_{1}\right)}\right), \ldots, p_{a\left(x_{n}\right)}^{-1}\left(U_{a\left(x_{n}\right)}\right)\right\}$ is an open cover of $p_{a}^{-1}\left(x_{a}\right)$. Let $b \geq a(x), a\left(x_{1}\right), \ldots, a\left(x_{n}\right)$ and let $U_{b}=$ $\cup\left\{p_{a\left(x_{1}\right) b}^{-1}\left(U_{a\left(x_{1}\right)}\right), \ldots, p_{a\left(x_{n}\right) b}^{-1}\left(U_{a\left(x_{n}\right)}\right)\right\}$. It follows that $p_{b}^{-1}\left(U_{b}\right) \subset U$ and $p_{a b}^{-1}\left(x_{a}\right) \subset U_{b}$. From being closed $p_{a b}$ it follows that there is an open set $U_{a}$ containing $x_{a}$ such that $p_{a b}^{-1}\left(U_{a}\right) \subset U_{b}$. Finally, $p_{a}^{-1}\left(U_{a}\right) \subset U$.

The following Theorem is the main result of this section.

Theorem 3.5. Let $\mathbf{X}=\left\{X_{a}, p_{a b}, A\right\}$ be an inverse system of non-empty $S(n)$-closed spaces and $\Theta^{n}$-closed bonding mapping $p_{a b}$. Then $\lim \mathbf{X}$ is nonempty. Moreover, if $p_{a b}$ are surjections, then the projections $p_{a}: \lim \mathbf{X} \rightarrow X_{a}$, $a \in A$, are surjections. 
Proof. Consider the following diagram

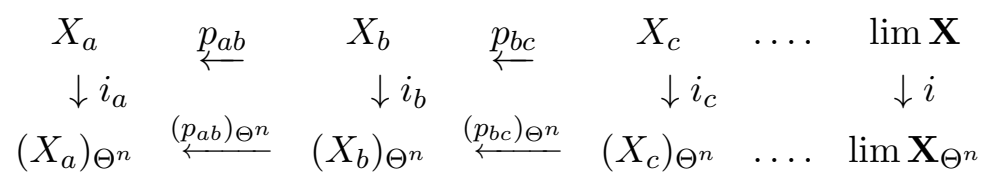

from Proposition 13. By Theorem 2.12 each $\left(X_{a}\right)_{\Theta^{n}}$ is a quasi-compact $T_{1}$ space. Furthermore, each mapping $\left(p_{a b}\right)_{\Theta^{n}}$ is closed by c) of Lemma 2.4 since $p_{a b}$ is $\Theta^{n}$-closed (see Definition 2.8). This means that the inverse system $\mathbf{X}_{\Theta}=\left\{\left(X_{a}\right)_{\Theta},\left(p_{a b}\right)_{\Theta}, A\right\}$ satisfies the conditions of Theorem 3.2. It follows that $\lim \mathbf{X}_{\Theta}$ is non-empty. This implies that $\lim \mathbf{X}$ is non-empty. Further, if $p_{a b}, b \geq a$, are onto mappings, then for each $x_{a} \in X_{a}$ the sets $Y_{b}=p_{a b}^{-1}\left(x_{a}\right)$ are non-empty $\Theta^{n}$-closed sets (Lemma 2.8). This means that the system $\mathbf{Y}_{\Theta}=\left\{\left(Y_{b}\right)_{\Theta},\left(p_{b c}\right)_{\Theta} \mid\left(Y_{c}\right)_{\Theta}, a \leq b \leq c\right\}$ satisfies Theorem 3.2 and has a non-empty limit. This means $\mathbf{Y}=\left\{Y_{b}, p_{b c} \mid Y_{c}, a \leq b \leq c\right\}$ has a non-empty limit. For every $y \in Y$ we have $p_{a}(y)=x_{a}$.

Lemma 3.6. Let $\mathbf{X}=\left\{X_{a}, p_{a b}, A\right\}$ be an inverse system of $S(n)$-closed spaces and $\Theta^{n}$-closed surjective bonding mapping $p_{a b}$. Then the projections $p_{a}: \lim \mathbf{X} \rightarrow \mathbf{X}_{a}, a \in A$, are $\Theta^{n}$-closed if and only if the mapping $p_{\Theta}$ : $(\lim \mathbf{X})_{\Theta^{n}} \rightarrow \lim \mathbf{X}_{\Theta^{n}}$ from Proposition 14 is a homeomorphism.

Proof. The "if" part. Now we have the following diagram

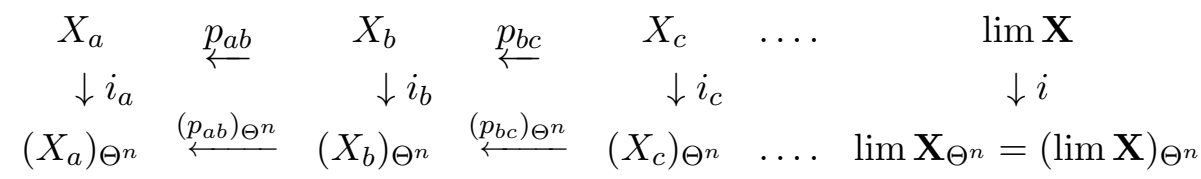

where $i$ and each $i_{a}$ is the identity for every $a \in A$. Each projection $q_{a}$ : $(\lim \mathbf{X})_{\Theta^{n}} \rightarrow\left(X_{a}\right)_{\Theta^{n}}$ is closed (Lemma 3.4). From (a) and (c) of Lemma 2.4 it follows that the projection $p_{a}: \lim \mathbf{X} \rightarrow \mathbf{X}_{a}$ is $\Theta^{n}$-closed for every $a \in A$.

The only "if" part. Suppose that the projections $p_{a}: \lim \mathbf{X} \rightarrow \mathbf{X}_{a}, a \in A$, are $\Theta^{n}$-closed. Let us prove that $p_{\Theta}$ is a homeomorphism. It suffice to prove that $p_{\Theta}$ is closed. Let $F \subset(\lim \mathbf{X})_{\Theta^{n}}$ be closed. This means that $F$ is $\Theta^{n}$-closed in $\lim \mathbf{X}$. For each $a \in A$ the set $p_{a}(F)$ is $\Theta^{n}$-closed since the projections $p_{a}$ are $\Theta^{n}$-closed. Now, $i_{a} p_{a}(F)$ is closed in $\left(X_{a}\right)_{\Theta^{n}}$. We have the collection $\left\{q_{a}^{-1} i_{a} p_{a}(F): a \in A\right\}$ having the finite intersection property. It is clear that $p_{\Theta}(F)=\cap\left\{q_{a}^{-1} i_{a} p_{a}(F): a \in A\right\}$ and that $\cap\left\{q_{a}^{-1} i_{a} p_{a}(F)\right.$ : $a \in A\}$ is closed in $\lim \mathbf{X}_{\Theta^{n}}$. Hence, $p_{\Theta}$ is closed and, consequently, a homeomorphism.

Question. Let $\mathbf{X}=\left\{X_{a}, p_{a b}, A\right\}$ be an inverse system of $\mathrm{S}(\mathrm{n})$-closed spaces Under what conditions $\lim \mathbf{X}$ is $\mathrm{S}(\mathrm{n})$-closed?

Now we shall prove some partial answers to this question. 
Theorem 3.7. Let $\mathbf{X}=\left\{X_{a}, p_{a b}, A\right\}$ be an inverse system of non-empty $S(n)$-closed spaces such that $\theta^{n}$-closure $C l_{\theta^{n}} M$ is Kuratowski Closure Operator i.e. $C l_{\theta^{n}}\left(C l_{\theta^{n}}(A)\right)=C l_{\theta^{n}}(A)$. If the projections $p_{a}: \lim \mathbf{X} \rightarrow \mathbf{X}_{a}, a \in$ $A$, are $\Theta^{n}$-closed, then $X=\lim \mathbf{X}$ is non-empty and $S(n)$-closed.

Proof. Using Theorem 3.5 we infer that $\lim \mathbf{X}$ is non-empty. By the only if part of Lemma 3.6 we infer that the mapping $p_{\Theta}:(\lim \mathbf{X})_{\Theta^{n}} \rightarrow \lim \mathbf{X}_{\Theta^{n}}$ from Proposition 14 is a homeomorphism, i.e. that $\lim \mathbf{X}_{\Theta^{n}}=(\lim \mathbf{X})_{\Theta^{n}}$. From Theorem 3.3 it follows that $\lim \mathbf{X}_{\Theta^{n}}$ is quasi-compact. Moreover, from $\lim \mathbf{X}_{\Theta^{n}}=(\lim \mathbf{X})_{\Theta^{n}}$ we infer that $(\lim \mathbf{X})_{\Theta^{n}}$ is quasi-compact. Finally, (a) of Theorem 2.12 completes the proof.

Theorem 3.8. If $\mathbf{X}=\left\{X_{a}, p_{a b}, A\right\}$ is an inverse system of non-empty $S(n)$-closed spaces $X_{a}$ and $H J$ mappings $p_{a b}$ such that $\theta^{n}$-closure $C l_{\theta^{n}} M$ is Kuratowski Closure Operator i.e. $C l_{\theta^{n}}\left(C l_{\theta^{n}}(A)\right)=C l_{\theta^{n}}(A)$, then $X=$ $\lim \mathbf{X}$ is non-empty and $S(n)$-closed.

Proof. Using Theorem 3.5 we infer that $\lim \mathbf{X}$ is non-empty. Then from Theorem 3.1 it follows that the projections $p_{a}: \lim \mathbf{X} \rightarrow \mathbf{X}_{a}, a \in A$, are HJ mapping and, consequently, $\Theta^{n}$-closed. Theorem 3.7 completes the proof.

Corollary 3.9. If $\mathbf{X}=\left\{X_{a}, p_{a b}, A\right\}$ is an inverse system of non-empty $S(n)$-closed spaces $X_{a}$ and semi-open (open, closed irreducuble) mappings $p_{a b}$ such that $\theta^{n}$-closure $C l_{\theta^{n}} M$ is Kuratowski Closure Operator i.e. Cl $l_{\theta^{n}}$ $\left(C l_{\theta^{n}}(A)\right)=C l_{\theta^{n}}(A)$, then $X=\lim \mathbf{X}$ is non-empty and $S(n)$-closed.

We say that an inverse system $\mathbf{Y}=\left\{Y_{a}, q_{a b}, A\right\}$ is a subsystem of $\mathbf{X}=$ $\left\{X_{a}, p_{a b}, A\right\}$ if $Y_{a} \subset X_{a}, a \in A$, and $q_{a b}=p_{a b} \mid Y_{b}$. In this case we shall write $\mathbf{Y}=\left\{Y_{a}, p_{a b} \mid Y_{b}, A\right\}$

Proposition 15. Let $\mathbf{X}=\left\{X_{a}, p_{a b}, A\right\}$ be an inverse system of non-empty $S(n)$-closed spaces and $\Theta^{n}$-closed bonding mapping $p_{a b}$. If $\mathbf{Y}=\left\{Y_{a}, p_{a b} \mid\right.$ $\left.Y_{b}, A\right\}$ is an inverse subsystem of $\Theta^{n}$-closed subsets $Y_{a} \subset X_{a}, a \in A$, then $\lim \mathbf{Y}$ is non-empty.

Proof. Now $\left\{\left(p_{a}\right)_{\Theta^{n}}^{-1} i_{a}\left(Y_{a}\right): a \in A\right\}$ is a family of closed sets in $\lim \mathbf{X}_{\Theta^{n}}$ with the finite intersection property. From the quasi-compactness of $\lim \mathbf{X}_{\Theta^{n}}$ (Theorem 3.3) it follows that $\cap\left\{\left(p_{a}\right)_{\Theta^{n}}^{-1} i_{a}\left(Y_{a}\right): a \in A\right\}$ is non-empty. Hence $\lim \mathbf{Y} \neq \emptyset$ since $\lim \mathbf{Y}=\mathbf{i}^{-1}\left(\cap\left\{\left(p_{a}\right)_{\Theta^{n}}^{-1} i_{a}\left(Y_{a}\right): a \in A\right\}\right)$.

\section{REFERENCES}

[1] M. P. Berri, J. R. Porter and R.M. Stephenson, A Survey of Minimal Topological Spaces, in: General Topology and its Relations to Modern Analysis and Algebra, Proc. Kanpur Top. Conf. (Academic Press, New York, 1970), 93-114. 
[2] R. F. Dickman, Jr., and Jack R. Porter, $\theta$-perfect and $\theta$-absolutely closed functions, Illinois J. Math., 21 (1977), 42-60.

[3] D. Dikranjan and E. Giuli, $S(n)-\theta$-closed spaces, Topology and its Applications 28 (1988), 59-74.

[4] R. Engelking, General Topology, PWN, Warszawa, 1977.

[5] T. Hamlett, H-closed spaces and the associated $\theta$-convergence space, Math. Chronicle, 8 (1979), 83-88.

[6] H. Herrlich, $T_{\nu}$-Abgeschlossenheit und $T_{\nu}$-Minimalititat, Math. Z., 88 (1965), 285294.

[7] J. Mioduszewski and L. Rudolf, H-closed and extremally disconnected Hausdorff spaces, Diss. Math., 51 (1969).

[8] M. N. Mukherjee and Atasi Debily, On H-closed space and grills, An. Ştiin. Univ. Al. I. Cuza Iaşi, Mat., 44 (1998), 1-25.

[9] A. V. Osipov, Nearly H-closed spaces, J. Math. Sci., 155 (2008), 624-631.

[10] J. R. Porter and C. Votaw, $S(\alpha)$ spaces and regular Hausdortf extensions, Pacific J. Math., 45 (1973), 327-345.

[11] J. R. Porter and J. Thomas, On H-closed and minimal Hausdorff spaces, Trans. Amer. Math. Soc., 138 (1969), 159-170.

[12] C. T. Scarborough, Minimal Urysohn spaces, Pacific J. Math., 27 (1968), 611-617.

[13] A. H. Stone, Inverse limits of compact spaces, Gen. Topology Appl., 10 (1979), 203211.

[14] L. Stramaccia, $S(n)$-spaces and H-sets, Comment. Math. Univ. Carolinae, 29 (1988), $221-226$.

[15] V. M. Uljanov, O bikompaktnyh rasširenijah sčetnogo haraktera i absoljutah, Mat. Sbornik, 98 (1975), 223-254.

[16] H. V. Velichko, H-closed topological spaces, Mat. Sb. (N.S.), 70 (112) (1966), 98-112.

[17] G. A. Viglino, $\bar{T}_{n}$ spaces, Notices Amer. Math. Soc., 16 (1969), 846.

(Received: August 1, 2014)

(Revised: December 3, 2014)
Faculty of Organizations and Informatics

Varaždin

Croatia

ivanloncar18@gmail.com 